\title{
3. METHODS
}

\section{Data sources}

\section{The New South Wales Midwives Data Collection}

The New South Wales Midwives Data Collection (MDC) is a population-based surveillance system covering all births in NSW public and private hospitals, as well as home births. It encompasses all live births and stillbirths of at least 20 weeks gestation or at least 400 grams birth weight.

The MDC relies on the attending midwife or doctor to complete a notification form when a birth occurs. The form, a copy of which is shown at Appendix 4, includes demographic items and items on maternal health, the pregnancy, labour, birth, and perinatal outcomes. Completed forms are sent to the Data Collections and Reporting Unit in the Demand and Performance Evaluation Branch of the NSW Department of Health, where they are compiled into the MDC database. About two-thirds of notifications are submitted electronically from hospital obstetric information systems to the MDC on-line system.

The MDC receives notifications of women whose usual place of residence is outside NSW but who give birth in NSW. However, the MDC does not receive notifications of births outside NSW to women usually resident in NSW.

\section{The Neonatal Intensive Care Units' Data Collection}

The Neonatal Intensive Care Units' (NICUS) Data Collection is a state-wide audit of infants admitted to neonatal intensive care units and 4 of the level 5 neonatal nurseries in New South Wales (NSW) and the Australian Capital Territory (ACT) during the neonatal period for one of the following reasons:

- gestational age less than 32 weeks;

- birth weight less than or equal to 1,500 grams;

- mechanical ventilation for 4 hours or more;

- continuous positive airways pressure (CPAP) for 4 hours or more;

- major surgery (opening of a body cavity);

- insertion of a central venous line for 4 hours or more.

In 2006 the ten neonatal intensive care units in NSW and ACT were situated at the following perinatal centres: John Hunter Children's Hospital—Newcastle, Liverpool Health Service, Nepean Hospital, Royal Hospital for Women, Royal North Shore Hospital, Royal Prince Alfred Hospital, The Canberra Hospital - Canberra, Westmead Hospital, and at the two paediatric hospitals: Sydney Children's Hospital and The Children's Hospital at Westmead. The four level five neonatal nurseries that joined NICUS between 2002 and 2005 were situated at Blacktown Hospital, Campbelltown Hospital, Gosford Hospital, St George Hospital and Wollongong Hospital. Unfortunately St George Hospital has been unable to provide data since 2004.

The neonatal, maternal and perinatal data which comprise the NICUS Data Collection are collected and collated within each neonatal intensive care unit and level five nursery by a designated Clinical Audit Officer. The data are compiled into a central database located at the NSW Centre for Perinatal Health Services Research.

\section{The Neonatal Intensive Care Units' Follow-up Data} collection

The Neonatal Intensive Care Units' Follow-up Data Collection is a state-wide audit at 2-3 years of age, corrected for prematurity of infants born 22 to 28 weeks gestation and admitted to a neonatal intensive care unit in NSW and the ACT.

All surviving infants were assessed by a developmental assessment team at the following perinatal centres: John Hunter Children's Hospital (Newcastle), Liverpool Health Service, Nepean Hospital, Royal Hospital for Women, Royal North Shore Hospital, Royal Prince Alfred Hospital, The Canberra Hospital (Canberra), Westmead Hospital or at one of the two paediatric hospitals: The Children's Hospital at Westmead, and the Sydney Children's Hospital.

The follow up data that comprise the NICUS Follow-up Data Collection are collected and collated within each of the above hospitals by a designated member of the developmental assessment team. The data are compiled into a central database located at the NSW Centre for Perinatal Health Services Research.

\section{The New South Wales Birth Defects Register}

The NSW Birth Defects Register (BDR) is a populationbased surveillance system established to monitor birth defects detected during pregnancy or at birth, or diagnosed in infants up to one year of age. The BDR was established in 1990 and, under NSW Public Health Act 1991, from 1 January 1998 doctors, hospitals, and laboratories have been required to notify birth defects detected during pregnancy, at birth, or up to one year of life. The BDR is administered by the Centre for Epidemiology and Research of the NSW Department of Health.

The activities of the BDR include: annual publication of information on birth defects in NSW; provision of information to area health services to assist in service planning and monitoring of child health, and investigation of specific issues; provision of information in response to specific requests from the public, health professionals, and other government departments; and provision of data to the AIHW National Perinatal Statistics Unit (NPSU) for monitoring of birth defects at a national level. The NPSU is also responsible for providing Australian information on birth defects to the International Clearinghouse for Birth Defects Monitoring Systems, a non-governmental organisation of the World Health Organization.

Sources of notifications to the BDR include: the NSW Midwives Data Collection (MDC), specialist paediatric 
hospitals, cytogenetic laboratories, and individual health care providers. The BDR is supported by an advisory committee, comprising a panel of clinical experts representing the following specialities: genetics, dysmorphology, neo-natology, obstetrics and gynaecology, midwifery, bioethics, and epidemiology; and a community representative from the Association of Genetic Support of Australasia.

Data for research purposes may be provided in 2 formats: aggregate information similar to that contained in this report, and data concerning individuals with identifying information removed. All requests for data should be submitted in writing to the Director, Centre for Epidemiology and Research. Requests for data concerning individuals for sufficiently important research purposes will be referred to the NSW Department of Health Ethics Committee. Procedures for release of personal information are described in the Department's Policy Directive PD2006-077 Data Collections - Disclosure of unit record data held for research or management of health services which is available on the NSW Department of Health's website at www.health.nsw.gov.au.

\section{The NSW Inpatient Statistics Collection}

For this report data from the NSW Admitted Patient Data Collection (APDC) was linked to MDC data to produce information on postnatal length of stay in NSW hospitals and mother's health insurance status.

The APDC covers demographic and episode related data for every inpatient who is separated from any public, private, and repatriation hospital, private day procedure centre, or public nursing home in NSW. Separation can result from discharge, transfer, death, or change in service category. The APDC is maintained by the Data Collections and Reporting Unit in the Demand and Performance Evaluation Branch of the NSW Department of Health.

\section{NSW Maternal and Perinatal Committee}

The NSW Maternal and Perinatal Committee is a quality assurance committee established under the NSW Health Administration Act 1982, and is privileged under the Act to carry out confidential reviews of both maternal and perinatal deaths. Members are appointed by the Minister for Health.

The Committee reviews each maternal death to identify any possible avoidable factors and to determine whether the death was related to pregnancy (or its management) or whether it was incidental. The Committee also reviews perinatal deaths among live born babies and stillbirths of at least 20 weeks gestation or at least 400 grams birth weight. The information obtained from these reviews assists in the development of policies aimed at improving the health of mothers and newborns in NSW. Information considered by the Committee is confidential.

\section{Method for estimating level of reporting of maternal Aboriginality}

The Aboriginality of the mother, rather than the baby, is reported to the MDC, although mother's Aboriginality is frequently used as a proxy measure for the baby's Aboriginality. Consequently, maternal Aboriginality was used for this analysis. The number of births reported to Torres Strait Islander mothers is quite small in NSW. Aboriginal and Torres Straight Islander mothers were therefore combined for this analysis. For ease of reference, 'Aboriginal' is used to refer to both Aboriginal or Torres Strait Islander mothers.

Records of births reported to the MDC were linked to birth registration records of the NSW Registry of Births, Deaths and Marriages for births occurring in the 4-year period 2002-2005. Record linkage was carried out at the Centre for Health Record Linkage (www.cherel.org.au). The overall linkage rate was 91.0 per cent of MDC records and 98.5 per cent of birth registration records.

Capture-recapture methods are used to adjust estimates of counts to reflect ascertainment level or undercounting. Capture-recapture was carried out using the method described by McCarty et al. ${ }^{1}$ Analysis was carried out using SAS version 9.1.3. Analyses concerning geographic location were based on health area of residence as reported to the MDC.

\section{References}

1. McCarty DJ, Tull ES, Moy CS, Kwoh CK, LaPorte RE. Ascertainment corrected rates: Applications of Capture-Recapture Methods. Int J Epidemiol 1993; 22(3): 559-565.

\section{Definitions}

\section{Aboriginal and Torres Strait Islander}

Women who identify themselves to be of Australian Aboriginal and Torres Strait Islander heritage.

\section{Age corrected for prematurity}

Age corrected for prematurity: until the child is 3 years old, the age of the baby is calculated from the due date and not the date of birth.

\section{Apgar score}

A numerical scoring system routinely administered one and 5 minutes after birth to evaluate the condition of the baby. The score ranges from $0-10$ (10 being perfect). It takes account of 5 physical signs, each of which is assigned a component score of 0,1 or 2 : heart rate, respiration, muscle tone, reflexes, and colour.

\section{Augmentation}

Artificial rupture of the membranes or use of oxytocic drugs after spontaneous onset of labour.

\section{Birth defect}

Any structural defect or chromosomal abnormality detected during pregnancy, at birth, or in the first year of life, excluding birth injuries and minor anomalies such as skin tags, talipes, birthmarks, or clicky hips. From 1994, the following conditions were included in the NSW Birth Defects Register: congenital hypothyroidism, cystic fibrosis, phenylketonuria, and thalassaemia major. 


\section{Birth weight}

The newborn infant's first bare weight in grams. Low birth weight: birth weight less than 2,500 grams.

Very low birth weight: birth weight less than 1,500 grams. Extremely low birth weight: birth weight less than 1,000 grams.

\section{Caesarean section}

Birth of the fetus through an abdominal incision. Elective caesarean section: a caesarean section (planned or unplanned) performed before the onset of labour. Emergency caesarean section: a caesarean section performed after the onset of labour, whether or not the onset of labour was spontaneous.

\section{Confinement}

Refers to a woman having given birth. In a multiple pregnancy, one confinement will result in more than one birth.

\section{Epidural}

Injection of analgesic agent outside the dura mater which covers the spinal canal; includes lumbar, spinal, and epidural anaesthetics.

\section{Episiotomy}

An incision of the perineum and vagina to enlarge the vulval orifice.

\section{Functional disability: ${ }^{1}$}

None-minimal: No developmental delay: Griffiths 7 Mental Developmental Scales (GMDS) general quotient (GQ) or Bayley Scales of Infant Development-II (BSID-II) mental developmental index (MDI) 1 standard deviation below the mean to 3 standard deviations above the mean;

Mild: Developmental delay: GMDS or BSIDII between 1 and 2 standard deviations below the mean; Mild cerebral palsy: able to walk without aids at 2-3 years of age, corrected for prematurity;

Moderate: Developmental delay: GMDS or BSIDII between 2 and 3 standard deviations below the mean; Moderate cerebral palsy: able to walk with the assistance of aids at 2-3 years of age, corrected for prematurity; Sensorineural or conductive deafness: requiring amplification with bilateral hearing aids or unilateralbilateral cochlear implant;

Severe: Developmental delay: GMDS or BSIDII 3 or more standard deviations below the mean; Bilateral blindness: with a visual acuity of $<6 / 60$ in the better eye; Severe cerebral palsy: unable to walk with the assistance of aids at 2-3 years of age, corrected for prematurity.

\section{Gestational age}

The duration of pregnancy in completed weeks from the first day of the last normal menstrual period. Where accurate information on the date of the last menstrual period is not available, a clinical estimate of gestational age may be obtained from ultrasound during the first half of pregnancy or by examination of the newborn infant. The 'best estimate' is used here.

\section{Griffiths Mental Developmental Scales (GMDS)}

The mean for the General Quotient (GQ) of the Griffiths Mental Developmental Scales (GMDS) is 100.2 and the standard deviation (SD) is 12.8 .

The mean for the mental developmental index (MDI) of the Bayley Scales of Infant Development-II (BSID-II) is 100 and the standard deviation (SD) is 15 .

\section{Induction of labour}

Oxytocics-prostaglandins: the initiation of labour by the use of oxytocic agents, prostaglandins, or their derivatives (oral, intravaginal or intravenous).

ARM only: the initiation of labour by artificial rupture of membranes.

Oxytocics-prostaglandins and ARM: both medical and surgical induction as defined above (combined medical and surgical induction).

\section{Intraventricular haemorrhage (IVH)}

Worst level of intraventricular haemorrhage (IVH) seen on either right or left side by either ultrasound or postmortem examination.

None: ultrasound-post-mortem shows no haemorrhage

Grade 1: subependymal germinal matrix haemorrhage

Grade 2: intraventricular haemorrhage with no ventricular dilatation

Grade 3: intraventricular haemorrhage with ventricle distended with blood

Grade 4: intraparenchymal haemorrhage not examined: No ultrasound or post-mortem examination

\section{Live birth}

The complete expulsion or extraction from its mother of a baby of at least 400 grams or 20 weeks gestation who, after being born, breathes or shows any evidence of life such as a heartbeat.

\section{Major surgery}

Any surgery that requires opening of a body cavity.

\section{Mechanical ventilation}

Use of a mechanical ventilator to provide intermittent positive pressure respiration for a baby for 4 hours or more.

\section{Necrotising enterocolitis (NEC)}

Clinically diagnosed: received treatment for NEC (includes suspending feeds, blood cultures and treatment with antibiotics such as clindamycin-gentamycin).

Proven radiologically or at operation: radiological signs include intra-mural or intra-hepatic air, perforation or a 'fixed loop'.

\section{Neonatal death}

The death of a live born infant within 28 days of birth. 


\section{Neonatal period}

The first 28 completed days of life.

\section{Neonatal mortality rate}

The number of neonatal deaths per 1,000 live births.

\section{Patent ductus arteriosus (PDA)}

Clinical signs of PDA such as typical murmur, active precordium, bounding pulses, cardiomegaly, or pulmonary vascular congestion on x-ray. May be confirmed on ultrasound examination.

\section{Parity}

The total number of live births and stillbirths of the mother before the pregnancy or birth under consideration.

\section{Perinatal death}

A stillbirth or neonatal death.

\section{Perinatal mortality rate}

The number of perinatal deaths (stillbirths and neonatal deaths) per 1,000 total births in a year (live births and stillbirths combined).

\section{Perineal status}

1st degree tear: a perineal graze-laceration-tear involving: the fourchette, hymen, labia, skin, vagina, or vulva.

2nd degree tear: a perineal laceration or tear involving the pelvic floor or perineal muscles or vaginal muscles.

3rd degree tear: a perineal laceration-tear involving the anal sphincter or rectovaginal septum.

4th degree tear: a third degree perineal laceration or tear which also involves the anal mucosa or rectal mucosa.

\section{Plurality}

The number of fetuses or babies from the pregnancy. On this basis pregnancy may be classified as single or multiple.

\section{Premature infant}

An infant born before 37 completed weeks gestation.

\section{Premature labour}

The spontaneous onset of labour (regular painful contractions with progressive cervical changes) before 37 completed weeks of gestation.

\section{Retinopathy of prematurity}

Worst stage of retinopathy of prematurity (ROP) in either eye during the initial hospital admission.

None seen: no changes seen

Stage I: demarcation line present

Stage II: ridge present

Stage III: ridge with extra-retinal fibrovascular proliferation

Stage IV: retinal detachment

Systemic infection in the infant

Clinical or radiological signs of infection together with growth of a known pathogen from a systemic site - does not include tracheal aspirate.

\section{Transfer (NICUS only)}

Maternal transfer before birth (prenatal): the transfer of a pregnant woman to a tertiary obstetric hospital. Neonatal transfer after birth (postnatal): the transfer of an infant from the hospital of birth to a tertiary NICU.

\section{Spontaneous abortion}

The spontaneous expulsion of a fetus less than 20 weeks gestation and less than 400 grams birth weight.

\section{Stillbirth}

The complete expulsion or extraction from its mother of a product of conception of at least 20 weeks gestation or 400 grams birth weight who did not, at any time after birth, breathe, or show any evidence of life such as a heartbeat.

\section{Termination of pregnancy}

A procedure intentionally performed to terminate a pregnancy before 20 completed weeks gestation.

\section{References}

1. The Victorian Infant Collaborative Study Group. Postnatal corticosteroids and sensorineural outcome at 5 years of age. J Paediatr Child Health 2000; 36:256-261.

\section{Explanatory notes}

\section{Antenatal complications (NICUS)}

These specifically include antepartum haemorrhage, placenta praevia, placenta abruptio, prolonged rupture of membranes, gestational diabetes, threatened preterm labour, hypertensive disease of pregnancy and rhesus isoimmunisation. There is also an open-ended 'other antenatal complications' option. The most common problems specified in this option are cervical incompetence, polyhydramnios, oligohydramnios, chorioamnionitis, threatened miscarriage, and problems secondary to multiple pregnancy.

\section{Rates of birth defects}

The BDR collects data pertaining to birth defects regardless of the outcome of pregnancy. This includes notifications of live births, stillbirths, terminations of pregnancy and spontaneous abortions. Birth defect rates are calculated using births (that is, live births and stillbirths) as the denominator, because denominator populations for pregnancies less than 20 weeks gestation are unknown. The numerators are described in the relevant sections.

The source of denominator population data on births is the MDC. The MDC was selected because its definitions are consistent with those applied by the BDR. Denominator populations compatible with the BDR were derived from the MDC by including only those births that occurred to NSW residents.

Caution should be exercised when comparing the birth defect rates tabled in this document with those reported in the AIHW National Perinatal Statistics Unit's Congenital Anomalies in Australia Report or interstate birth defects 
registers. Variations may be due to differences in coding practices, in categories of birth defects included in each Register and differences in the upper age limit for notification of cases.

\section{Place of residence of mother}

The mother's usual residence was the basis for coding to statistical local areas and NSW health areas.

\section{Labour}

The category labour-spontaneous with oxytocicsprostaglandins was used where labour was augmented with artificial rupture of membranes as well as oxytocics or prostaglandins.

\section{Levels of neonatal care}

\section{Tertiary}

Level 3: Neonatal Intensive Care Unit (NICU) - a unit that provides high-dependency specialist nursing and medical care for all newborn infants including sustained life support such as mechanical ventilation and has staff neonatologists and neonatal registrars.

\section{Non-tertiary}

Level 2a: Neonatal Care-a unit that can give high-level oxygen, can start mechanical ventilation if necessary, and has paediatric house staff.

Level 2b: Neonatal Care-a unit that can give low-level oxygen and has a paediatrician on call.

\section{Level of maternity hospital}

Level 1: local maternity service (no births), postnatal only for women with normal outcomes.

Level 2: small maternity services, normal-risk pregnancy and births only. Staffed by general practitioners and midwives.

Level 3: country district and smaller metropolitan services, care for mothers and infants at normal - selected moderate risk pregnancies and births. Full resuscitation and theatre facilities available. Rostered obstetricians, resident medical staff and midwives. Accredited general practitioners and specialist anaesthetist on call. Has Level $2 b$ neonatal care.

Level 4: regional - referral - metropolitan district services. Birth and care for mothers and/or babies with moderate risk factors. Obstetricians and paediatricians available 24 hours a day, 7 days a week. Rostered resident medical staff, specialist anaesthetist on call. Has Level $2 \mathrm{~b}$ neonatal care.

Level 5: regional referral - metropolitan services, care for mothers and infants known to be at high risk. Able to cope with complications arising from these risk factors. Has Level 2a neonatal care.

Level 6: (tertiary) - specialist obstetric services (supra regional). All functions - normal, moderate and high-risk births. Has Level 3 neonatal intensive care.

\section{Type of birth}

The 'vaginal breech' category covers all forms of vaginal breech birth, including forceps to the after-coming head.

\section{Perinatal mortality rate}

Perinatal deaths include deaths reported to the MDC only. As the MDC form is completed at discharge or transfer of the baby, deaths occurring after this time may not be reported to the MDC. Birth and perinatal death registration data held by the Australian Bureau of Statistics (ABS) give the most complete ascertainment of perinatal deaths for calculation of rates. 


\section{MAP OF NSW HEALTH AREAS}
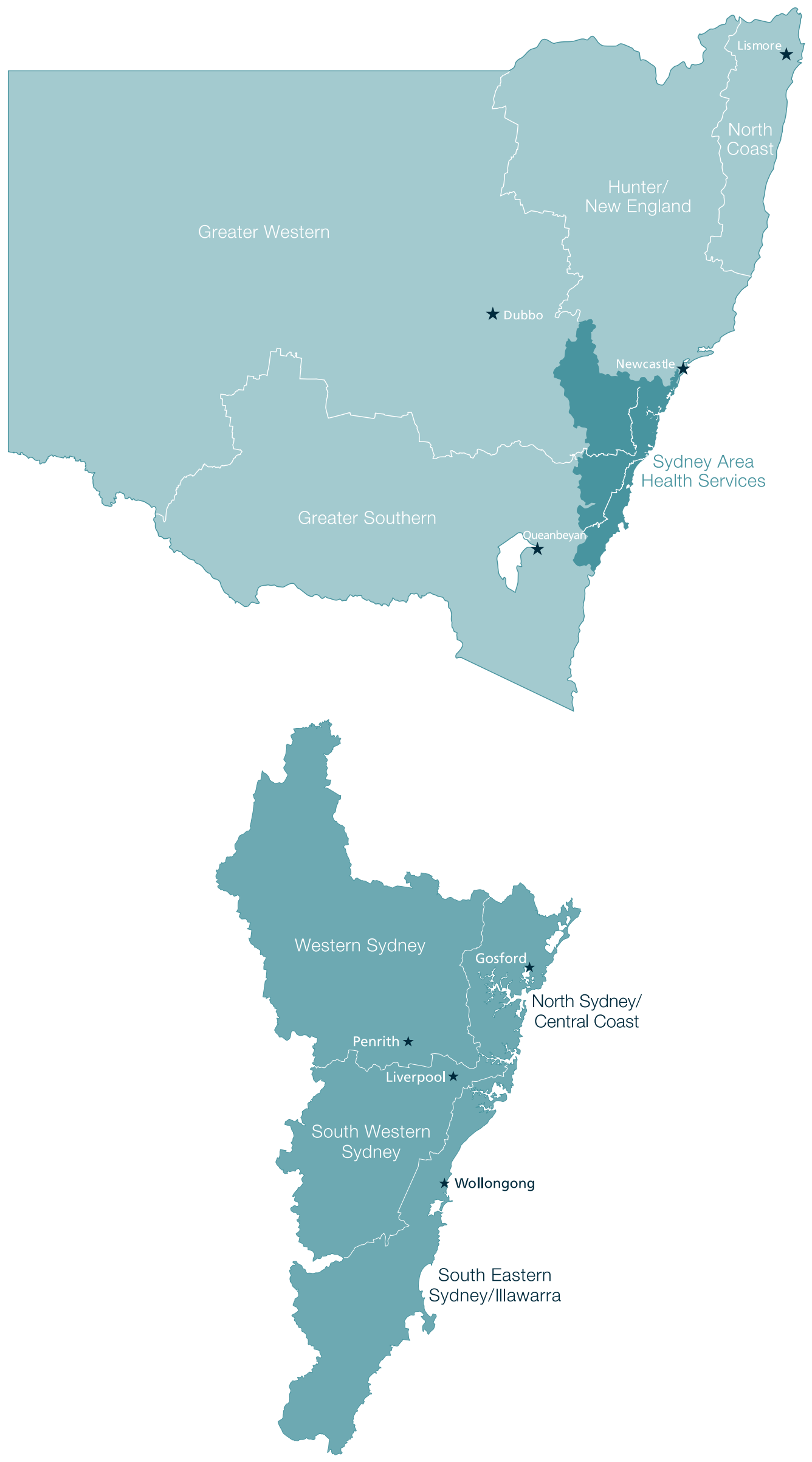